\title{
Biologia do caranguejo-uçá, Ucides cordatus (Crustacea: Decapoda: Brachyura), na Baía da Babitonga, Santa Catarina, Brasil
}

\author{
Alison C. Wunderlich ${ }^{\text {; }}$ Marcelo A. A. Pinheiro ${ }^{1} \&$ Ana Maria T. Rodrigues ${ }^{2}$ \\ 1 Universidade Estadual Paulista, Campus Experimental do Litoral Paulista, Grupo de Pesquisa em Biologia de Crustáceos. \\ Praça Infante Dom Henrique, 11330-900 São Vicente, São Paulo, Brasil. E-mail: crusta@csv.unesp.br; pinheiro@csv.unesp.br \\ ${ }_{2}$ Instituto Chico Mendes de Conservação da Biodiversidade, Centro de Pesquisa e Gestão de Recursos Pesqueiros do Litoral \\ Sudeste e Sul. Avenida Ministro Victor Konder, 88301-700 Itajaí, Santa Catarina, Brasil.
}

\begin{abstract}
Biology of the mangrove uçá crab, Ucides cordatus (Crustacea: Decapoda: Brachyura), in Babitonga Bay, Santa Catarina, Brazil. The aim of this work is to characterize, for the first time, aspects of the reproduction of the mandrove-land crab in mangroves of the Babitonga Bay (Santa Catarina). Furthermore, density and stock size of this fishery resource were also estimated. The specimens were sampled from May 2002 to April 2003, from two distinct locations: Iperoba and Palmital; a total of 2265 specimens (1623 males and 642 females) were analyzed. Males with mature gonads were recorded throughout the year, while females with mature gonads were only recorded during a five-month period. Ovigerous females were recorded in December and January. The ethogram of the reproductive migration phenomenon ("andada") is in agreement of high crab activity associated to full- and new-moon, with higher intensity during December and January, also related to the austral summer season. The total sampling density on Iperoba Mangrove was $2.05 \pm 0.97$ individuals. $\mathrm{m}^{-2}$ and did not differ significantly $(\mathrm{p}<0.05)$ from the density recorded on Palmital Mangrove $\left(2.06 \pm 1.08\right.$ individuals. $\left.\mathrm{m}^{-2}\right)$. The overall average of density estimation at Babitonga Bay was $2.05 \pm 1.00$ individuals. $\mathrm{m}^{-2}$, which corresponded to the density of open $(1.42 \pm$ 0.89 individuals. $\left.\mathrm{m}^{-2}\right)$ and closed burrows $\left(0.64 \pm 0.63\right.$ individuals. $\left.\mathrm{m}^{-2}\right)$.

KEY WORDS. Andada; fishery; reproduction; sampling density.
\end{abstract}

RESUMO. O objetivo deste estudo é caracterizar pela primeira vez alguns aspectos da reprodução do caranguejouçá em manguezais da Baía da Babitonga (Santa Catarina). Além disso, a densidade e o tamanho do estoque deste recurso pesqueiro foram também estimados. Os exemplares foram coletados mensalmente, de maio de 2002 a abril de 2003, em duas áreas distintas: Iperoba e Palmital; um total de 2265 espécimes (1623 machos e 642 fêmeas) foi analisado. Os machos com gônadas maturas foram registrados durante todo o ano, enquanto as fêmeas com gônadas maturas ocorreram em apenas cinco meses. As fêmeas ovígeras foram registradas apenas em dezembro e janeiro. O etograma do fenômeno de migração reprodutiva ("andada") esteve em concordância com a maior atividade de caranguejos associada às luas cheias e novas, com maior intensidade em dezembro e janeiro, relacionados ao verão austral. A densidade total no Manguezal de Iperoba foi de $2,05 \pm 0,97 \mathrm{ind} . / \mathrm{m}^{2}$, não diferindo significativamente daquela registrada para o Manguezal do Palmital $\left(2,06 \pm 1,08\right.$ ind./ $\left.\mathrm{m}^{2}\right)(\mathrm{p}<0,05)$. A média global para a estimativa de densidade na Baia da Babitonga foi de $2,05 \pm 1,00$ ind. $/ \mathrm{m}^{2}$, correspondendo a $1,42 \pm$ 0,89 ind. $/ \mathrm{m}^{2}$ com base nas galerias abertas e $0,64 \pm 0,63$ ind. $/ \mathrm{m}^{2}$ para as galerias fechadas.

PALAVRAS-CHAVE. Andada; densidade; pesca; reprodução.

Ucides cordatus (Linnaeus, 1763) é uma espécie de caranguejo semiterrestre exclusiva de áreas de manguezal, com distribuição no Atlântico Ocidental, da Flórida (EUA) até Laguna, Santa Catarina (Brasil) (Melo 1996). Possui expressiva importância econômica no litoral brasileiro (Fausto-Filho 1968, JANKowsky et al. 2006), servindo como fonte de alimento e renda para um grande contingente humano que se ocupa de sua extração e comércio (Ibama 1994, Glaser \& Diele 2004, Passos \& Di BeneditTo 2005). Além disso, desempenha também importante papel ecológico no ecossistema manguezal, atuando no processamento da serrapilheira (NordHAus et al. 2006), no fluxo energético (Conde et al. 2000, Wolf et al. 2000), na bioturbação do sedimento (Amouroux \& TAVAres 2005), e na ciclagem do carbono e da matéria orgânica (Guest et al. 2006). 
Desde 1998, o Centro de Pesquisa e Gestão de Recursos Pesqueiros do Litoral Sudeste e Sul (CEPSUL/ICM-Bio), integrante do recém criado Instituto Chico Mendes de Conservação da Biodiversidade (anteriormente integrante do Instituto Brasileiro de Meio Ambiente) vem promovendo reuniões de ordenamento pesqueiro com abrangência regional. Estas reuniões envolvem um Grupo Gestor, constituído por representantes do poder público, da academia e do setor produtivo, que estruturam através de gestão compartilhada as regras de uso para o Recurso Caranguejo-Uçá. O objetivo destes eventos é congregar informações sobre a espécie visando à elaboração de normativa para uso deste recurso nas regiões Sudeste e Sul do Brasil (Rodrigues et al. 2000).

Embora as pesquisas sobre a biologia e ecologia desta espécie tenham se intensificado nos últimos anos, ainda são escassos os estudos que tratam da sua dinâmica reprodutiva e densidade (Branco 1993, Blankensteyn et al. 1997, Pinheiro \& Fiscarelli 2001, Diele et al. 2005). Para o estabelecimento da legislação de ordenamento pesqueiro, o estudo da dinâmica reprodutiva é de extrema importância, pois orienta a tomada de decisões mais adequadas ao manejo do recurso. A determinação da época reprodutiva de crustáceos pleociematos tem sido freqüentemente delimitada pelos meses de ocorrência de fêmeas ovígeras e/ou com gônadas maturas na população, durante um período anual (SASTRY 1983, Dalabona \& Loyola-Silva 2005). De acordo com a classificação proposta por PINHeiro \& Fransozo (2002), a duração da época reprodutiva desses crustáceos pode ser dividida em três categorias: 1) contínua; 2) sazonal; e 3) sazonal-contínua. A caracterização do padrão reprodutivo de uma espécie é ferramenta fundamental como auxílio à gestão de um recurso pesqueiro, particularmente do caranguejo-uçá, por sua importância comercial.

A compreensão dos comportamentos reprodutivos também é primordial ao melhor embasamento das normativas propostas. Como exemplo, destaca-se o comportamento migratório de alguns crustáceos durante as épocas de acasalamento, cópula e liberação larval (HARTNOLl 1969, Bliss 1990), quando estão mais vulneráveis à captura. Neste sentindo, entre os crustáceos já estudados destacam-se a lagosta Palinurus argus (Latreille, 1804) (veja BrusCA \& BRUSCA 2003); o gecarcinídeo Gecarcoidea natalis (Pocock, 1888) (veja Debeluus 1999); e alguns portunídeos, como Callinectes sapidus (Rathbun, 1896) e Scylla olivacea (Herbst, 1794) (veja Clark et al. 1999 e KoolKalya et al. 2006, respectivamente).

A migração de $U$. cordatus é conhecida popularmente por "andada" ou "carnaval do caranguejo" (AlCÂNTARA-FilHo 1978, Costa 1979). Nesta ocasião, todos os indivíduos desta espécie abandonam suas galerias e caminham ativamente sobre o sedimento (NAscimento 1993), os machos liberam espuma e lutam com outros machos para a atração/posse das fêmeas (PINHEIRO \& Fiscarelli 2001), que então são copuladas e, posteriormente, liberam suas larvas na água dos estuários (Andrea Freire com. pes.) ou da própria galeria (Péricles Góes com. pes.). Neste mo- mento, há uma intensificação da exploração comercial deste recurso pela maior facilidade de captura, como também pelo aumento de sua procura para consumo humano, particularmente nos meses de verão (Glaser \& Diele 2004).

A densidade é outro parâmetro imprescindível ao manejo do caranguejo-uçá, embora os estudos já realizados não tenham tomado precauções para minimização do erro amostral (BRANCO 1993, Blankensteyn et al. 1997, Ivo et al. 2000, Alves \& Nishida 2004, Alves et al. 2005, Diele et al. 2005). A estimativa da densidade de caranguejos semiterrestres tem sido um tópico relativamente bem abordado na literatura (WARREN 1990, LOURENÇO et al. 2000, FLores et al. 2005). No entanto, a escolha do método ideal para se obter uma estimativa confiável é um dos maiores entraves a estes estudos, particularmente para braquiúros semiterrestres que habitam costões rochosos (Flores \& PaUla 2002) e manguezais (NobBS \& McGuiness 1999, Macia et al. 2001, Skov et al. 2002). A quantificação direta do número de galerias/área tem sido um dos métodos mais indicados à estimativa da densidade populacional de braquiúros semiterrestres (LouRenço et al. 2000, Skov et al. 2002). A morfologia da abertura das galerias de $U$. cordatus, bem como sua inclinação de $45^{\circ}$ em relação ao sedimento (Costa 1979, Geraldes \& Calventi 1983), possibilitam seu fácil reconhecimento em relação às demais espécies escavadoras, particularmente das espécies de Uca. Tais estimativas para o caranguejo-uçá ganham ainda maior robustez quando as galerias abandonadas (sem atividade biogênica) são desconsideradas durante as análises e aquelas com dupla abertura contadas como uma única galeria (Gustavo Hattori com. pes.). Utilizando-se tal refinamento, os valores estimados têm sido muito próximos aos reais (BREITfuss 2003), trazendo grande aceitação da comunidade científica ao método de estimativa indireta da densidade por quantificação das galerias $/ \mathrm{m}^{2}$ (SKov et al. 2002, JoRDÃo \& Oliveira 2003).

Os estudos de densidade sobre o caranguejo-uçá na região sul (BRANCO 1993, Blankensteyn et al. 1997) têm evidenciado valores inferiores aos das regiões norte e nordeste do Brasil (Costa 1979, Alcântara-Filho 1978, Diele et al. 2005). Pelo exposto, a maior parte dos estudos sobre U.cordatus foram desenvolvidos na região equatorial, existindo uma carência de contribuições para o sul do Brasil, cuja importância se respalda na clina latitudinal que envolve os aspectos de ordem biológica e ecológica. Vale ressaltar, também, a ausência de estudos sobre a biologia desse caranguejo na Baía da Babitonga, o que justifica o esforço, tendo em vista: 1) o grande interesse na exploração sazonal deste crustáceo pela população local; e 2) localização desta região se encontrar próxima ao limite austral de ocorrência dos manguezais e, por conseguinte, da espécie.

O presente estudo visa analisar aspectos da biologia do caranguejo-uçá, principalmente aqueles relativos à delimitação da época reprodutiva (dinâmica gonadal e de fêmeas ovígeras na população), cópula (repleção da espermateca e fenômeno da "andada"), bem como da avaliação da densidade populacional e estimativa do estoque deste recurso nos manguezais da Baía da Babitonga (Santa Catarina), Brasil. 


\section{MATERIAL E MÉTODOS \\ Caracterização da área de estudos}

A Baía da Babitonga (Fig. 1) está situada no litoral norte de Santa Catarina $\left(26^{\circ} 02^{\prime} 00^{\prime \prime}-26^{\circ} 28^{\prime} 00^{\prime \prime}\right.$ S e $48^{\circ} 28^{\prime} 00^{\prime \prime}-48^{\circ} 50^{\prime}$ $00^{\prime \prime} \mathrm{W}$ ), totalizando uma área total de $130 \mathrm{~km}^{2}$ e possuindo em seu entorno a cidade de Joinville ( $\left.26^{\circ} 12^{\prime} 55^{\prime \prime} \mathrm{S}-49^{\circ} 20^{\prime} 16^{\prime \prime} \mathrm{S}\right)$ e de São Francisco do Sul $\left(26^{\circ} 19^{\prime} 52^{\prime \prime}\right.$ S- $48^{\circ} 20^{\prime} 53^{\prime \prime}$ S). A Baía da Babitonga pode ser dividida em três setores, sendo um deles compreendido pela baía propriamente dita (Setor Principal) e os outros dois por um canal que segue ao norte (Setor Palmital) e outro ao sul (Setor Linguado), confluindo na altura da Ilha do Mel ( $\left.26^{\circ} 17^{\prime} 54^{\prime \prime} S-48^{\circ} 44^{\prime} 31^{\prime \prime} S\right)$. Trata-se da maior área de manguezal existente em Santa Catarina, onde se concentra $75 \%$ do total desse ecossistema no estado, com área estimada em 6200 ha (Iвама 1998). O Setor Palmital possui a maior e mais exuberante área de manguezal $\left(45,37 \mathrm{~km}^{2}\right)$, enquanto que os demais possuem manguezais de área similar: 7,63 $\mathrm{km}^{2}$ (Setor Linguado) e $6,94 \mathrm{~km}^{2}$ (Setor Central).

No presente estudo foram selecionadas três áreas de manguezal (Fig. 1), sendo duas delas ( $\mathrm{P}=$ Palmital; e I = Iperoba) utilizadas para a análise biológica dos exemplares de $U$. cordatus, enquanto a terceira área $(\mathrm{C}=\mathrm{Capri})$ serviu ao registro diário do comportamento reprodutivo desta espécie. A área P (26 $16^{\circ} 52^{\prime \prime} \mathrm{S}$ $48^{\circ} 46^{\prime} 38^{\prime} \mathrm{W}$ ) dista $30 \mathrm{~km}$ da desembocadura da baía, conferindo-lhe maior influência continental; o bosque de manguezal apresenta predomínio de Avicennia schaueriana ( $\pm 8 \mathrm{~m}$ de altura), sedimento areno-lodoso com expressivo enraizamento e reduzida inundação (manguezal alto ou em barranco). A área I $\left(26^{\circ} 13^{\prime} 07^{\prime \prime} \mathrm{S}-48^{\circ} 35^{\prime} 07^{\prime \prime} \mathrm{W}\right)$, por estar cerca de $7 \mathrm{~km}$ da desembocadura da baia, recebe maior influência marinha; o bosque de manguezal possui predomínio de Laguncularia racemosa $( \pm 6 \mathrm{~m}$ de altura), sedimento lodoso pouco enraizado com maior inundação (manguezal baixo). A área C (26 $\left.11^{\prime} 12^{\prime \prime} S-48^{\circ} 33^{\prime} 45^{\prime \prime} \mathrm{W}\right)$, mais próxima da desembocadura da baia $(5 \mathrm{~km})$, apresenta bosque com predomínio de Laguncularia racemosa $( \pm 4 \mathrm{~m}$ de altura); o sedimento é areno-lodoso, com enraizamento e inundação intermediárias quando confrontado com as áreas P e I.

\section{Coleta e análise de dados}

Entre maio de 2002 a abril de 2003, foram realizadas coletas mensais da espécie, por "braceamento", na área de manguezal do Palmital e do Iperoba, sendo utilizados para as análises biológicas. Assim, para a dinâmica reprodutiva foram capturados 100 exemplares por área de manguezal (200 exemplares/mês). As galerias sofreram alargamento com "chuncho" (Portaria IBAMA \# 52/2003) e os caranguejos utilizados nas análises da maturidade gonadal e repleção da espermateca foram capturados manualmente por "braceamento", acondicionados em sacos plásticos e mantidos sob congelamento.

Após descongelados à temperatura ambiente, os animais foram sexados por inspeção da morfologia abdominal/número de pleópodes (Pinheiro \& Fiscarelli 2001) e classificados em três grupos de interesse (machos, fêmeas não ovígeras e fêmeas oví-

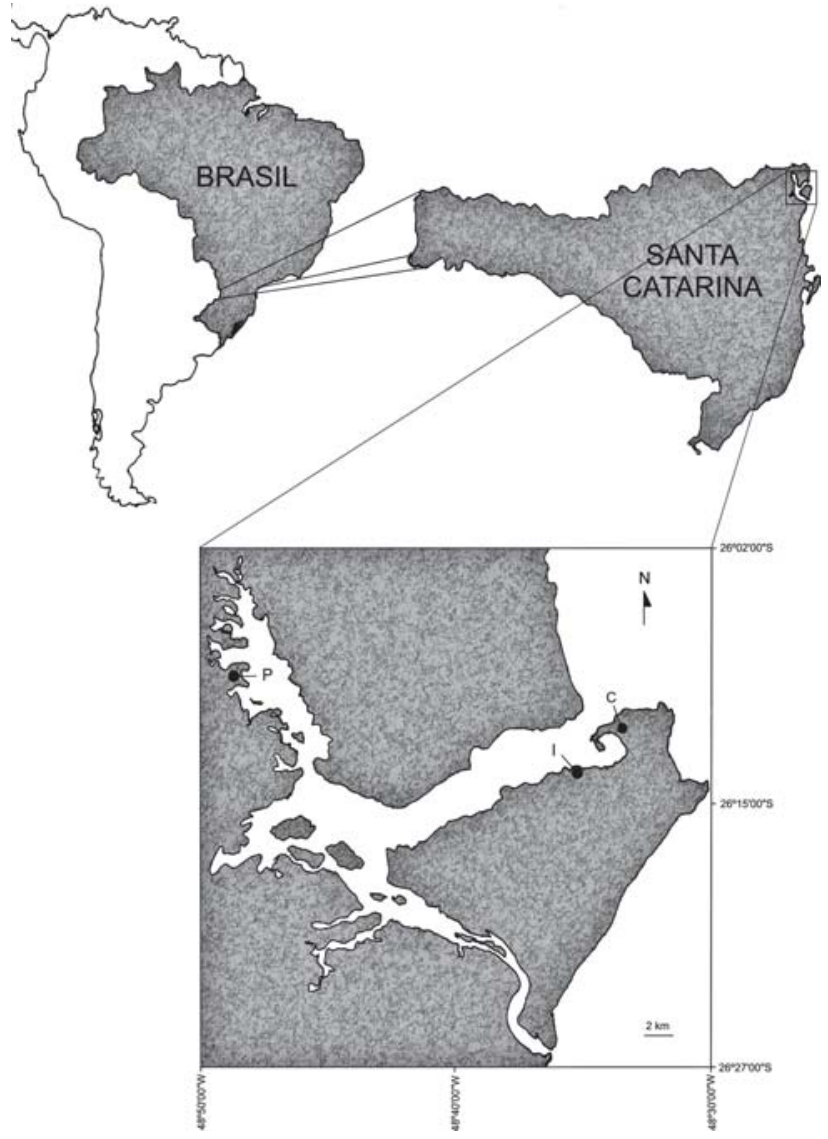

Figura 1. Localização das áreas de manguezal na Baía da Babitonga, Santa Catarina, onde foram realizadas as coletas mensais ( $\mathrm{I}=$ Iperoba, $\mathrm{P}=$ Palmital) e o registro da "andada" da espécie ( $\mathrm{C}=$ Capri $)$.

geras). Cada indivíduo teve a largura cefalotorácica (LC) medida com paquímetro $(0,05 \mathrm{~mm})$, enquanto o peso úmido total (PE) foi registrado em balança de precisão $(0,01 \mathrm{~g})$, após secagem prévia em papel absorvente.

Para determinar a época reprodutiva, os indivíduos foram dissecados e as gônadas categorizadas macroscopicamente por sua coloração e tamanho em relação ao hepatopâncreas, conforme recomendado por Күомо (1988). Assim, foram estabelecidos para cada sexo seis sub-estágios de maturação, agrupados, posteriormente, em três estágios (imaturo, I = sub-estágios 1 e 2; em maturação, EM = sub-estágios 3 e 4; e maturo, $\mathrm{M}=$ sub-estágios 5 e 6), conforme recomendado por PINHEIro \& Fiscarelli (2001). As fêmeas tiveram as espermatecas removidas e categorizadas em três estágios, segundo seu grau de repleção com espermatóforos (vazia: V, meiro-túrgida: MT, e túrgida: T), conforme utilizado por Pinheiro \& Fransozo (2002), adaptado para a espécie em questão.

A época reprodutiva de $U$. cordatus foi delimitada pelos meses de ocorrência de fêmeas ovígeras (FO\%) na população, 
sendo confrontada com o registro mensal de fêmeas com gônadas maturas, visando conferir maior fidedignidade à análise (Pinheiro \& Fransozo 2002). A freqüência relativa mensal dos três estágios de maturação gonadal de cada sexo, bem como dos três estágios de repleção da espermateca, foram utilizados na confecção de gráficos de área para análise de sua dinâmica.

Como durante o período da "andada" ocorre um aumento expressivo do número de espécimes do caranguejo-uçá caminhando sobre o sedimento dos manguezais, a delimitação deste período foi obtida pelos dias onde foram registradas as maiores intensidades. As observações foram realizadas no manguezal do Capri, por um período de quase quatro meses completos ( 5 de outubro de 2002 a 28 de fevereiro de 2003). Para este fim, uma área de observação de 10x10 m (100 m²) foi demarcada com fitas plásticas, onde os exemplares eram facilmente avistados e contados. A quantificação diária da "andada" foi padronizada pelo registro da abundância absoluta de indivíduos $/ \mathrm{m}^{2}$ durante a primeira maré baixa diurna, servindo de base à elaboração do etograma reprodutivo. Outros eventos, como a liberação de espuma e confrontos agonísticos entre machos, também foram registrados e associados às fases lunares, temperatura atmosférica e precipitação.

A densidade do caranguejo-uçá foi determinada mensalmente durante o período de estudos para a área do Iperoba (influência marinha) e do Palmital (influência de água doce). Para quantificar a densidade populacional em cada área foram utilizados cinco quadrados de amostragem ( $2 \times 2 \mathrm{~m})$, sendo o primeiro deles disposto a cinco metros da margem, e os demais contíguos ao interior do bosque de manguezal. A densidade da espécie foi estabelecida em cada quadrado pelo método indireto (número de galerias $/ \mathrm{m}^{2}$ ), quantificando apenas as galerias que apresentavam presença do animal, segundo três categorias: 1) galeria aberta com atividade biogênica, com acúmulo de lama fluida, fezes e rastros junto à abertura; 2) galeria fechada recente, cuja abertura apresentava-se ocluída por um "tampão" de sedimento úmido; e 3) galeria fechada antiga (ou "batumada"), quando a mesma não apresentava abertura visível, sendo reconhecida pela elevação e textura mais áspera do sedimento, sendo confirmada por escavação. As galerias abertas sem atividade biogênica, foram consideradas abandonadas e descartadas das análises, enquanto aquelas que apresentavam dupla abertura eram contadas como uma única galeria.

As galerias abertas com atividade biogênica tiveram o diâmetro de sua abertura (DG) medido com paquímetro $(0,05$ $\mathrm{mm})$, sempre tomados em paralelo à superfície do sedimento. Aquelas com DG > $45 \mathrm{~mm}$ foram consideradas ocupadas por exemplares com tamanho acima do mínimo estabelecido para captura (LC > $60 \mathrm{~mm}$ ), conforme conversão entre estas variáveis pela equação $C C=0,893 \mathrm{LC}^{0,957}\left(\mathrm{~N}=2130 ; \mathrm{r}^{2}=0,98\right)$, obtida por Pinheiro \& Hattori (2006). A densidade média mensal por área de manguezal resultou da somatória dos três tipos de galerias anteriormente citados, nos cinco quadrados de amostragem, com posterior divisão pela metragem quadrada dos mesmos $\left(20 \mathrm{~m}^{2}\right)$. A densidade de $U$. cordatus foi também estabelecida para cada tratamento (galerias abertas, fechadas e total de galerias), nas áreas do Iperoba e Palmital, sendo os dados submetidos a uma ANOVA, num delineamento inteiramente casualizado com mesmo número de repetições, com a comparação das médias realizada pelo Teste de Tukey $(\alpha=0,05)$.

O Potencial de Extração Imediato (PEI) do caranguejouçá na área do Iperoba e do Palmital foi obtido pelo cálculo da densidade de galerias com DG > $45 \mathrm{~mm}$, enquanto o Potencial de Extração Futuro (PEF) foi estabelecido pelos valores de densidade com DG inferiores a este tamanho. A diferença entre os percentuais de PEI e PEF foram analisadas pelo teste G (SoKAL \& ROHLF 2003).

\section{RESULTADOS}

Foram coletados 2265 exemplares de $U$. cordatus, correspondendo a 1623 machos e 642 fêmeas (625 não ovígeras e 17 ovígeras) (Tab. I). Os machos apresentaram média de LC superior à das fêmeas $(68,0 \pm 1,1>58,7 \pm 0,9 \mathrm{~mm} ; \mathrm{F}=447,4 ; \mathrm{p}<$ 0,05), o mesmo ocorrendo para a variável PE $(145,6 \pm 4,6>$ $86,2 \pm 3,9$ g; F $=514,2 ; p<0,05)$.

Tabela I. Abundância absoluta mensal dos morfotipos de $U$. cordatus durante o período de maio de 2002 a abril de 2003, nos manguezais de Iperoba e Palmital (Santa Catarina).

\begin{tabular}{|c|c|c|c|c|c|}
\hline \multirow{2}{*}{ Mês/ano } & \multirow{2}{*}{ Machos } & \multicolumn{2}{|c|}{ Fêmeas } & \multirow{2}{*}{ PO\% } & \multirow{2}{*}{ Total } \\
\hline & & Sem ovos & Ovígeras & & \\
\hline Mai/2002 & 138 & 63 & 0 & 0 & 201 \\
\hline Jun & 154 & 39 & 0 & 0 & 193 \\
\hline Jul & 151 & 51 & 0 & 0 & 202 \\
\hline Ago & 138 & 39 & 0 & 0 & 177 \\
\hline Set & 124 & 46 & 0 & 0 & 170 \\
\hline Out & 144 & 61 & 0 & 0 & 205 \\
\hline Nov & 137 & 63 & 0 & 0 & 200 \\
\hline Dez & 178 & 24 & 13 & 35,10 & 215 \\
\hline $\operatorname{Jan} / 2003$ & 126 & 60 & 4 & 6,30 & 190 \\
\hline $\mathrm{Fev}$ & 106 & 55 & 0 & 0 & 161 \\
\hline Mar & 119 & 69 & 0 & 0 & 188 \\
\hline $\mathrm{Abr}$ & 108 & 55 & 0 & 0 & 163 \\
\hline Total & 1623 & 625 & 17 & 2,65 & 2265 \\
\hline
\end{tabular}

A maturação gonadal da espécie foi avaliada para 2235 exemplares (1616 machos e 619 fêmeas). Os machos com gônadas maturas ocorreram durante todo o ano, com cinco incidências, sendo três delas mais expressivas (dezembro, 56,7\%; fevereiro, 71,4\%; e abril, 75,9\%) (Fig. 2), correspondendo aos meses de primavera (38\%) e verão (25\%) (Fig. 6). As fêmeas com gônadas maturas foram registradas em apenas cinco meses (novembro a março), com duas maiores incidências anuais (no- 

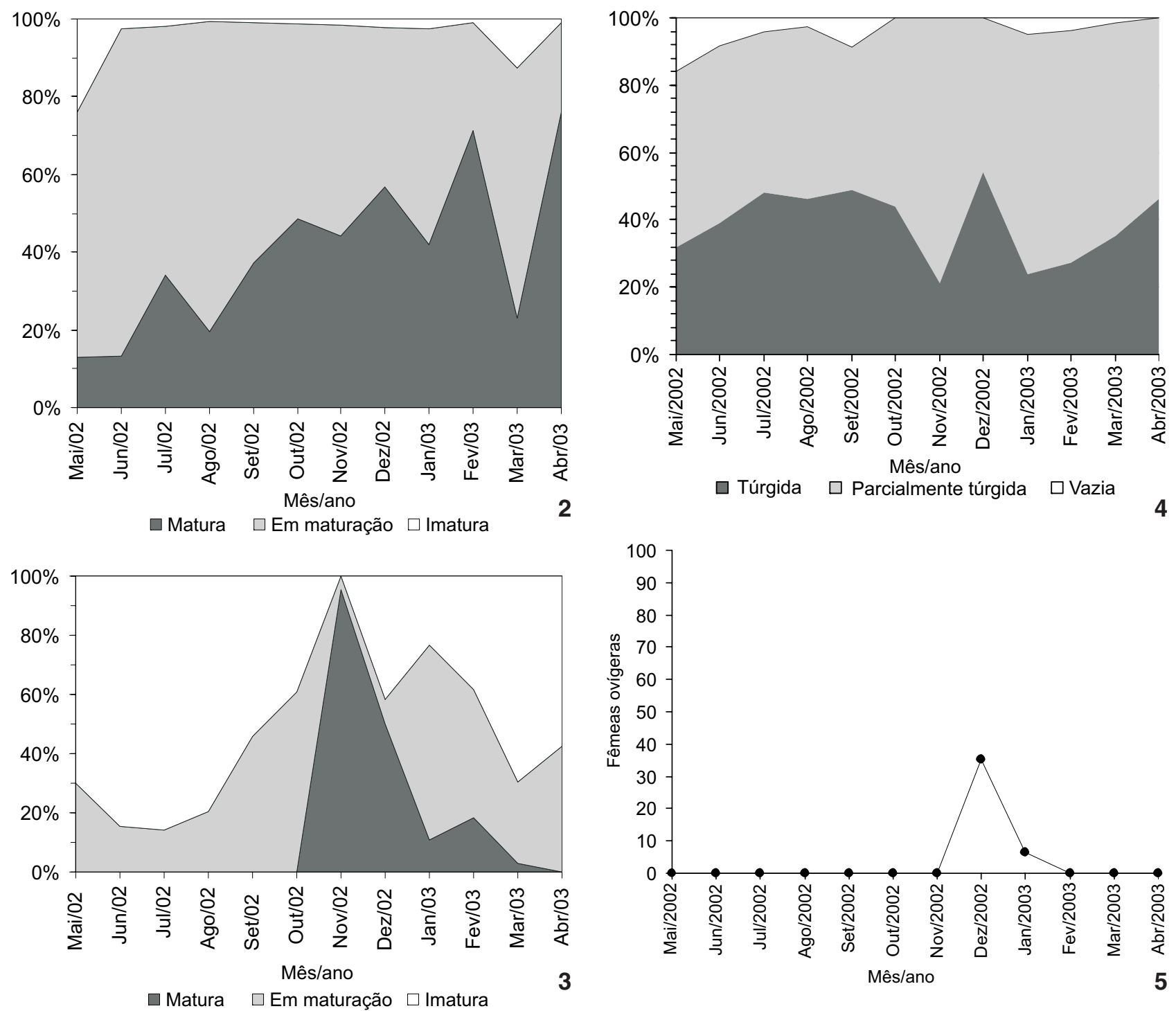

Figuras 2-5. (2-3) Dinâmica dos estágios gonadais de Ucides cordatus, com base no registro mensal para os machos (2) e fêmeas (3); (4-5) Dinâmica dos estágios de repleção da espermateca das fêmeas de U. cordatus (4), bem como percentual de fêmeas ovígeras em relação ao total de fêmeas capturadas mensalmente (5).

vembro, 95,2\%; e fevereiro, 18,2\%) (Fig. 3), também na primavera e verão (81 e 19\%, respectivamente) (Fig. 6).

Para a determinação do período de cópula foram analisadas 590 fêmeas durante o período de estudos. Aquelas com espermatecas túrgidas $(37,8 \%)$ foram registradas em todos os meses de amostragem (Fig. 4), embora com três incidências de cópula (julho-outubro, dezembro e abril), não tendo sido verificado contraste entre as estações do ano (Fig. 7) ( $p>0,05$ ).

As fêmeas ovígeras foram registradas somente em dezembro $(35,1 \%)$ e janeiro $(6,5 \%)$ (Fig. 5), correspondendo à primavera e verão $(\mathrm{p}<0,05)$, respectivamente (Fig. 8$)$.
O etograma de "andada" da espécie (Figs 9-11) evidencia que as maiores incidências estiveram associadas com as luas cheias e novas, ou mesmo dois a três dias depois das mesmas (Fig. 9). Os meses de maior intensidade da "andada" foram dezembro e janeiro (Fig. 13), não sendo verificada associação com a temperatura $(r=0,10 ; p>0,05)$ ou pluviosidade $(r=0,02 ; p>0,05)$.

$\mathrm{Na}$ figura 14 verifica-se um aumento do percentual de galerias fechadas (recentes + "batumadas") no período de agosto a outubro, bem como antecipação deste evento para a Área de Iperoba.

No Iperoba a média de densidade total foi de 2,05 $\pm 0,97$ 
ind./ $\mathrm{m}^{2}$, não diferindo estatisticamente da média observada para o Palmital (2,06 $\pm 1,08$ ind./ $\left.\mathrm{m}^{2}\right)$, a 5\% de significância (Tab. II). Desta forma, a média de densidade geral para a Baia da Babitonga foi estimada em $2,05 \pm 1,00$ ind. $/ \mathrm{m}^{2}$, sendo de 1,42 $\pm 0,89$ ind. $/ \mathrm{m}^{2}$ e de $0,64 \pm 0,63$ ind. $/ \mathrm{m}^{2}$, com base nas galerias abertas e fechadas, respectivamente.

Tabela II. Densidade mensal (ind. $/ \mathrm{m}^{2}$ ) de $U$. cordatus nas duas áreas de manguezal da Baía da Babitonga, Santa Catarina, durante maio de 2002 a abril de 2003, com base na contagem do número total de galerias (abertas e fechadas).

\begin{tabular}{|c|c|c|c|c|c|c|}
\hline \multirow{2}{*}{ Mês/ano } & \multicolumn{3}{|c|}{ Iperoba } & \multicolumn{3}{|c|}{ Palmital } \\
\hline & Abertas & Fechadas & Total & Abertas & Fechadas & Total \\
\hline Mai/2002 & 2,45 & 0,10 & 2,55 & 0,60 & 0,25 & 0,85 \\
\hline Jun & 1,40 & 0,35 & 1,75 & 1,90 & 1,25 & 3,15 \\
\hline Jul & 0,80 & 1,05 & 1,85 & 2,15 & 1,15 & 3,30 \\
\hline Ago & 0,20 & 0,55 & 0,75 & 0,00 & 1,25 & 1,25 \\
\hline Set & 1,05 & 1,40 & 2,45 & 0,85 & 1,40 & 2,25 \\
\hline Out & 0,75 & 1,00 & 1,75 & 1,35 & 2,50 & 3,85 \\
\hline Nov & 1,30 & 0,05 & 1,40 & 1,10 & 0,40 & 1,50 \\
\hline Dez & 1,85 & 0,00 & 1,85 & 2,65 & 0,00 & 2,65 \\
\hline $\operatorname{Jan} / 2003$ & 1,25 & 0,35 & 1,60 & 0,75 & 0,10 & 0,85 \\
\hline Fev & 2,65 & 0,00 & 2,65 & 0,50 & 0,10 & 0,60 \\
\hline Mar & 1,15 & 0,20 & 1,35 & 1,45 & 0,35 & 1,80 \\
\hline Abr & 3,80 & 0,80 & 4,60 & 2,05 & 0,65 & 2,70 \\
\hline Total & $\begin{array}{c}, 55 \pm \\
0,99 \\
\end{array}$ & $\begin{array}{c}0,49 \pm \\
0,47 \\
\end{array}$ & $\begin{array}{l}2,04 \pm \\
0,97 a^{*}\end{array}$ & $\begin{array}{c}1,28 \pm \\
0,79 \\
\end{array}$ & $\begin{array}{c}0,78 \pm \\
0,74 \\
\end{array}$ & $\begin{array}{l}2,06 \pm \\
1,08 \mathrm{a} \\
\end{array}$ \\
\hline
\end{tabular}

* $p>0,05$.

Foram analisadas 754 galerias de Ucides cordatus, sendo 413 para a área do Iperoba e 341 para o Palmital (Tab. III). Não houve diferença estatística entre o PEI do Iperoba $(77,7 \%)$ em relação ao PEI do Palmital $(69,8 \%)$ (p > 0,05), ocorrendo o mesmo em relação ao potencial de extração futuro (PEF) destas duas áreas (22,3 e 30,2\%, respectivamente). Portanto, para a Baía da Babitonga o PEI resultou em 74,1\% e o PEF em 25,9\%.

\section{DISCUSSÃO}

A época reprodutiva dos crustáceos apresenta grande diversificação inter e intraespecífica, variando principalmente em
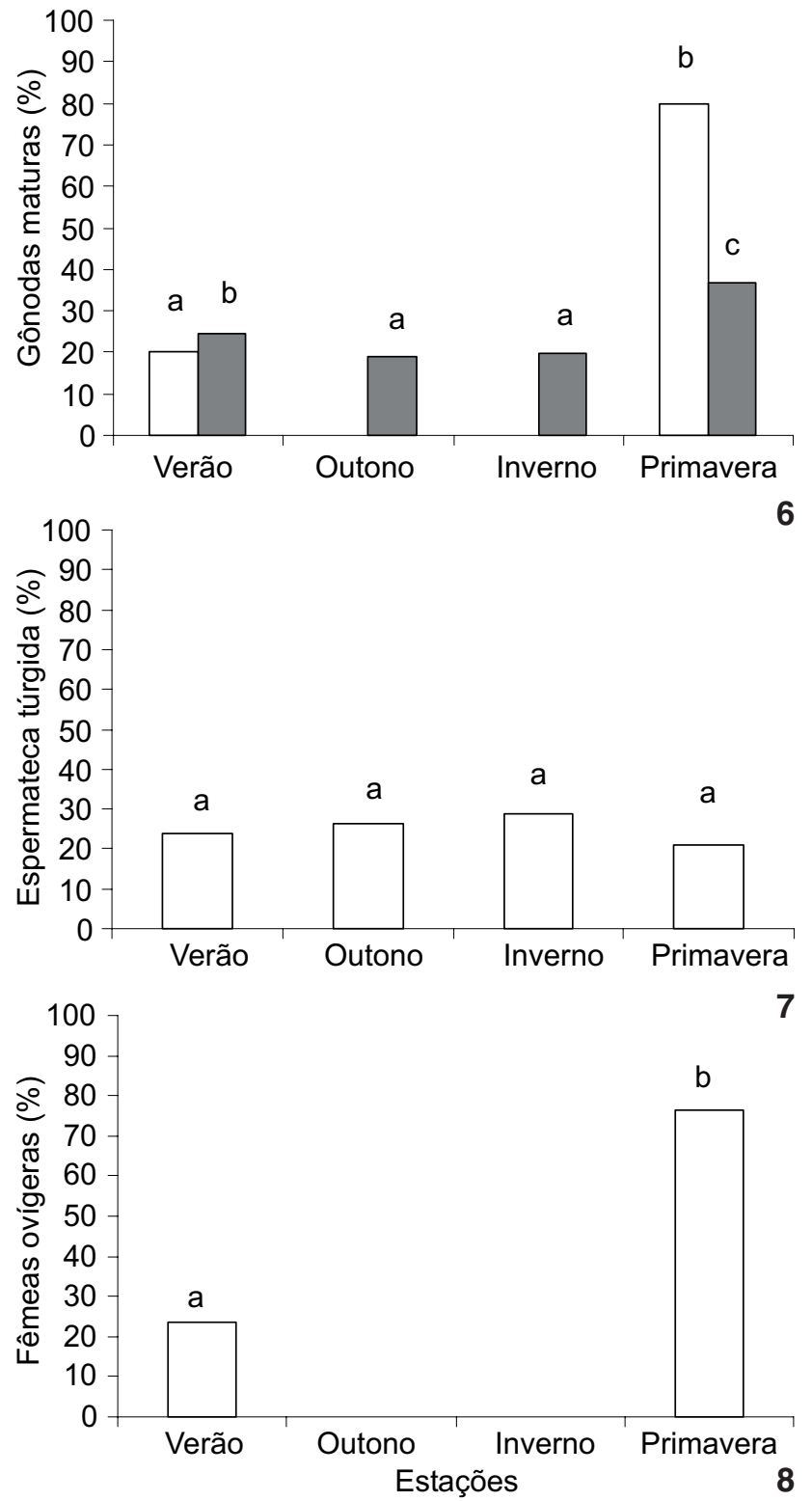

Figuras 6-8. Percentual sazonal de gônadas maturas dos machos (barras pretas) e fêmeas (barras brancas) de Ucides cordatus (6), das fêmeas com espermatecas túrgidas (7) e das fêmeas ovígeras em relação ao total de fêmeas capturadas (8).

Tabela III. Potencial extrativo imediato (DG $>45 \mathrm{~mm}$ ) e futuro $(\mathrm{DG}<45 \mathrm{~mm}$ ) de $U$. cordatus nas duas áreas de manguezal, sendo calculado com base no total de galerias abertas mensuradas. (DG) Diâmetro da galeria ( $\mathrm{mm}$ ).

\begin{tabular}{|c|c|c|c|c|c|}
\hline \multirow{2}{*}{ Manguezal } & \multicolumn{3}{|c|}{ Abundância de galerias } & \multicolumn{2}{|c|}{ Potencial extrativo (\%) } \\
\hline & $\mathrm{DG}<45 \mathrm{~mm}$ & $\mathrm{DG}>45 \mathrm{~mm}$ & Total & Futuro & Imediato \\
\hline Iperoba & 92 & 321 & 413 & 22,3 & 77,7 \\
\hline Palmital & 103 & 238 & 341 & 30,2 & 69,8 \\
\hline Geral & 195 & 559 & 754 & 25,9 & 74,1 \\
\hline
\end{tabular}



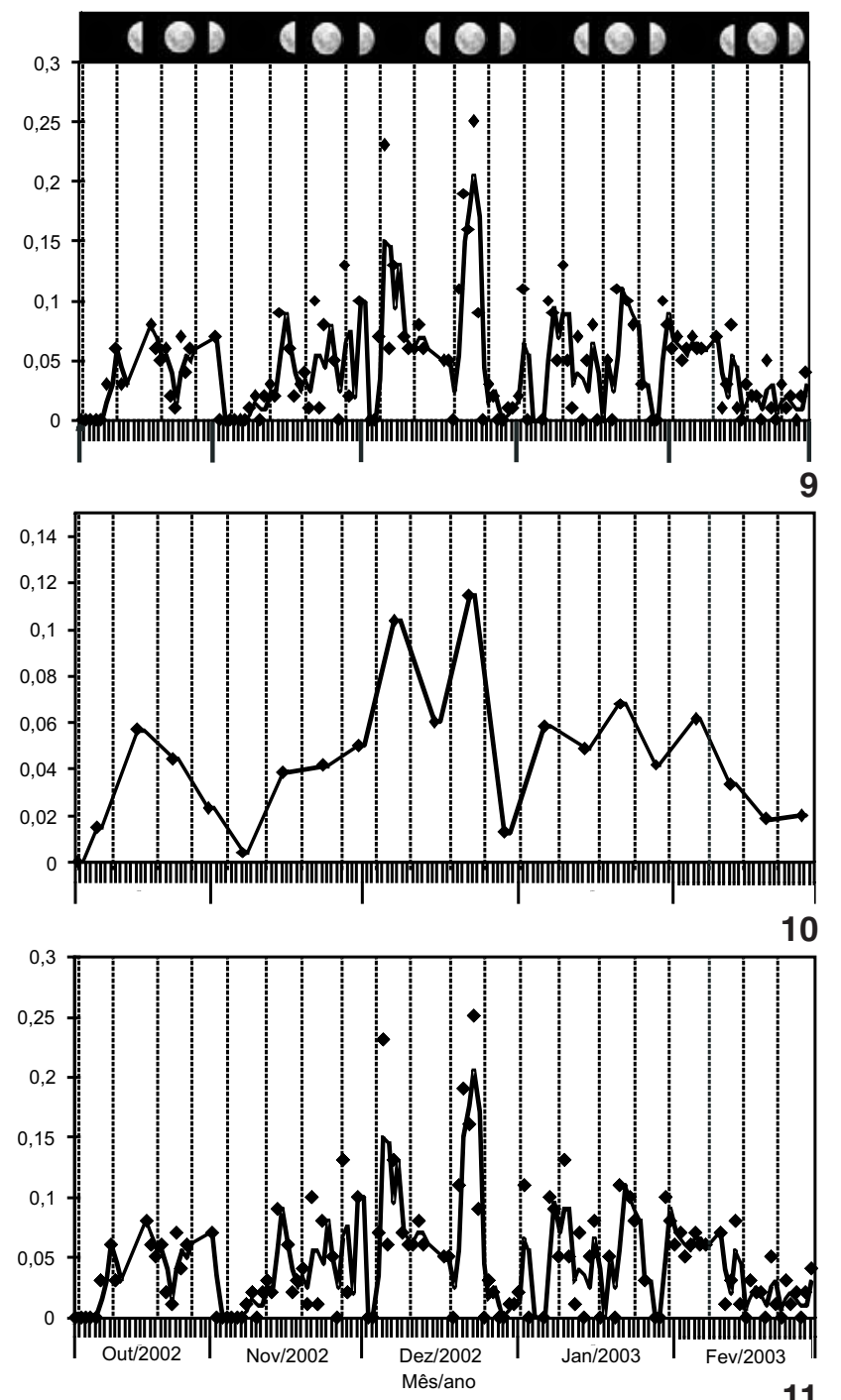

Figuras 9-11. Avaliação diária da densidade de indivíduos em atividade sobre o sedimento (andada) e nas fases lunares (9), média de densidade por fase lunar (10) e período de ocorrência de animais espumando (barras pretas) (11).

função dos fatores ambientais e da variação latitudinal (SASTRY 1983, Henmi 1993). Segundo SASTRY (1983), os crustáceos podem se reproduzir durante todos os meses do ano (padrão contínuo) ou se restringir a alguns meses onde as condições ambientais são mais favoráveis (padrão descontínuo ou sazonal). De acordo com este autor e Pinheiro \& Fransozo (2002), a reprodução de $U$. cordatus é do tipo sazonal, pois o registro de fêmeas com gônadas maturas ocorre somente em cinco meses do ano (novembro a março), englobando a ocorrência de fêmeas ovígeras (dezembro e janeiro). Tais informações são confirmadas pelos dados obtidos por Diele (2000), que mostra a restri-
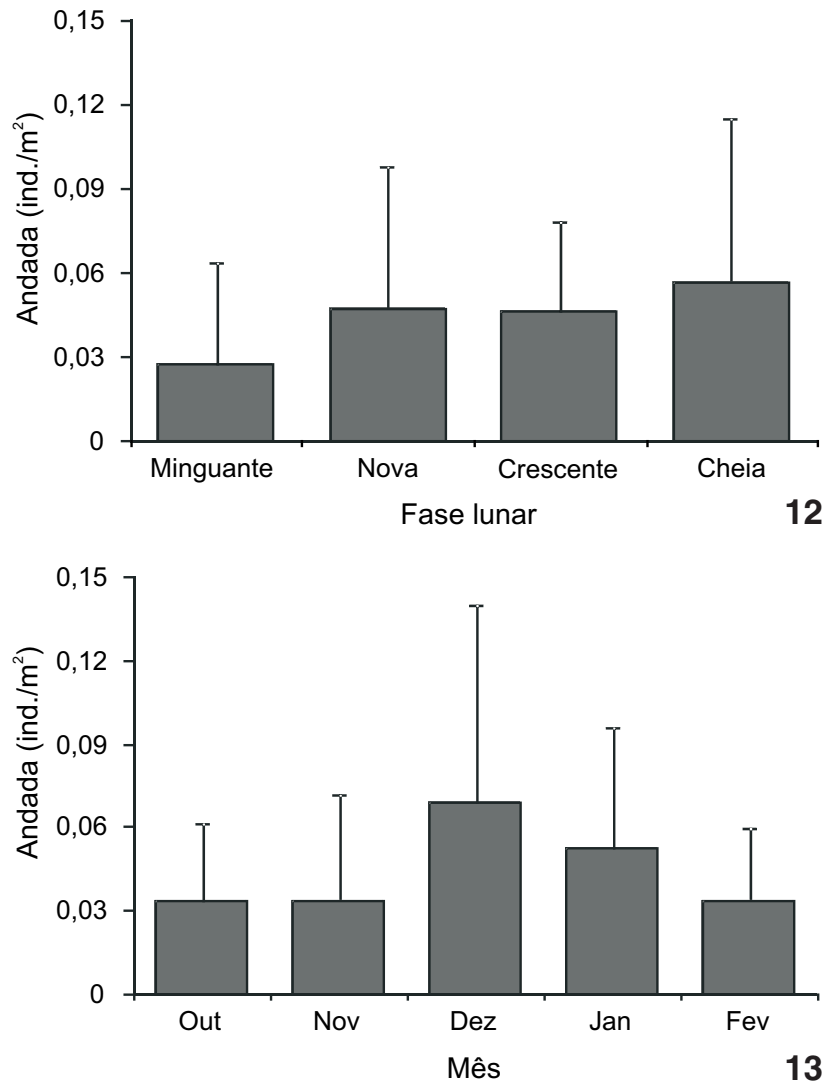

Figuras 12-13. Atividade locomotora de $U$. cordatus sobre o sedimento nas fases lunares (12) e nos meses (13). Barras = média, linha $=$ desvio padrão.

ção reprodutiva desta espécie à estação chuvosa, seguindo padrão similar ao de outros caranguejos semiterrestres e terrestres já estudados (Crane 1943, Haley 1972, Henmi 1989, Henmi \& Kaneto 1989, AdamczewsKa \& Morris 2001). Segundo MotaAlves (1975), a presença de machos com gônadas maturas durante todo o ano ocorre pela maior rapidez do ciclo espermiogênico em relação ao oogênico. Para as fêmeas de $U$. cordatus ocorreu associação do período reprodutivo (maturação gonadal e exemplares ovígeros) com a elevação da temperatura, fotoperíodo e precipitação, conforme já relatado por outros autores que estudaram esta espécie (Alcântara-Filho 1978, Costa 1979, Diele 2000, Pinheiro \& Fiscarelli 2001).

De acordo com PaYen (1980), a temperatura e o fotoperíodo são os principais parâmetros ambientais que regulam a reprodução nos crustáceos. Para $U$. cordatus, a redução térmica e de fotoperíodo parecem causar inibição da reprodução, com a maturação gonadal das fêmeas se iniciando somente a partir de agosto, quando ocorre a elevação destes parâmetros. Este processo é de extrema vantagem reprodutiva para $U$. cordatus, visto que sincroniza a ocorrência de fêmeas ovígeras com os meses de maior temperatura, bem como a eclosão das larvas 

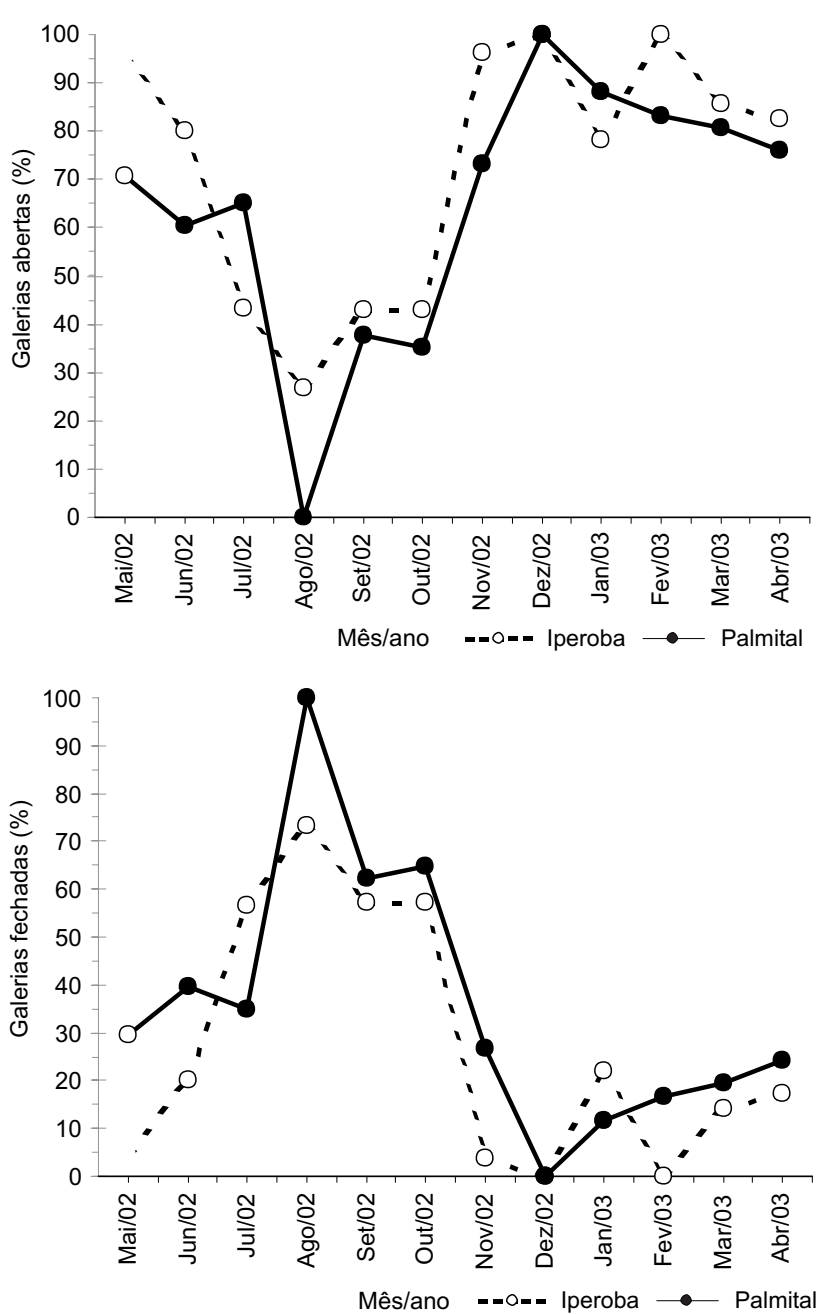

Figura 14. Percentual de galerias abertas e fechadas de Ucides cordatus na Baía da Babitonga, durante o período de maio de 2002 a abril de 2003.

com as maiores amplitude de marés, minimizando o tempo de desenvolvimento embrionário/larval e a predação na fase planctônica (Nye 1977, Lindley 1990ab, Pinheiro et al. 1994, Morgan 1996, Morgan \& Christy 1996).

O comportamento de "andada" de $U$. cordatus está intimamente ligado à época de acasalamento e liberação larval (PINHEIRO \& Fiscarelli 2001). Na época de acasalamento ocorre intensificação da atividade locomotora dos animais sobre o sedimento, devido à maior interação entre os machos pela posse das fêmeas (Fiscarelli \& Pinheiro 2002). Além disso, nesta ocasião é comum os machos do caranguejo-uçá liberarem espuma ao redor de seu corpo, próximo às galerias, facilitando seu reconhecimento pelas fêmeas, o que remete à importância dos estímulos visuais na atração sexual em caranguejos braquiúros (HaRTNoll 1969). A atividade de "andada" do caranguejo-uçá no manguezal da Baía da Babitonga foi influenciada pelo ritmo lunar, associada particularmente às luas cheias e novas, o que é corroborado pela literatura disponível (Costa 1979, AlCÂNTARA-Filho 1978, NASCIMENTO 1993, NORDI 1994). Em estudo realizado por NORDI (1994), em manguezais da Paraíba, este evento reprodutivo é associado às variações da maré em função das fases lunares. Este período coincidiu com o momento em que os animais liberavam espuma próximo às galerias, corroborando os dados de NASCIMENTO (1993), que constatou a liberação de espuma branca secretada pela boca dos caranguejos, antecedendo ao acasalamento. No presente estudo, não foram observados animais em confrontos, nem tampouco o acasalamento da espécie, o que pode ter ocorrido durante as marés de sizígia, que não foram avaliadas. Segundo Diele (2000) e Fiscarelli \& Pinheiro (2002), a "andada" de acasalamento ocorre entre dois a três dias depois da lua cheia ou nova, podendo perdurar até três dias. O presente estudo não constatou qualquer migração massiva de fêmeas ovígeras para as margens, o que pode ser explicado pela maior inundação do Manguezal do Capri, onde as larvas podem ser liberadas durante a maré alta, ou mesmo na água existente no interior de suas galerias (Péricles Góes com. pes.). Os dados obtidos também revelam que a desova desta espécie ocorreu em dezembro, cerca de um mês após a cópula (novembro), quando também ocorreu a maior incidência de fêmeas com gônadas maturas.

As estimativas de densidade dos caranguejos semiterrestres têm sido abordadas com certa freqüência na literatura (Warren 1990, Lourenço et al. 2000, Flores et al. 2005). Em função do modo de vida e do habitat ocupado por seus representantes, diversos métodos de amostragem têm sido avaliados para determinar com maior fidedignidade a densidade populacional (Macintosh 1988, Nobbs \& Mcguiness 1999, Skov \& Hartnoll 2001, Skov et al. 2002). Skov \& Hartnoll (2001) fizeram uma análise comparativa entre três métodos para a estimativa da densidade de Uca annulipes (H. Milne-Edwards, 1837), verificando que a contagem direta das galerias superestimava a densidade, embora representasse melhor o tamanho populacional. Assim, o método de estimativa indireta da densidade do caranguejo-uçá no presente estudo, pela quantificação do número de galerias/área, minimizou o viés amostral, em função da: 1) facilidade no reconhecimento das galerias desta espécie por sua morfologia externa e inclinação de $45^{\circ}$ em relação ao sedimento; 2) desconsideração do número de galerias abandonadas (sem atividade biogênica) nas análises; e 3) inspeção do número de aberturas/galeria da espécie, considerando-se aquelas com dupla abertura como uma única galeria. Concordando com Breitfuss (2003), tais procedimentos minimizam o erro deste método em caranguejos semiterrestres, maximizando cerca de três vezes sua precisão (33 para 97\%).

O tamanho do estoque populacional de $U$. cordatus foi determinado com base na premissa de que cada galeria comportava apenas um único exemplar. Tal afirmação encontra respaldo nos estudos realizados por Alcântara-FilHo (1978) e CosTA (1979), em manguezais na região nordeste brasileira. 
A redução na abundância de galerias abertas nas duas áreas de manguezal a partir de julho pode ser explicada pela redução térmica característica do inverno na região. Assim, a maior incidência de galerias fechadas nos meses de agosto e setembro coincide com o período de muda da espécie, confirmando os resultados de Pinheiro \& Fiscarelli (2001) para uma população dessa espécie em Iguape (São Paulo). A antecipação dos eventos de oclusão das galerias do caranguejo-uçá no manguezal de Iperoba, em comparação ao do Palmital, pode ser explicada pela maior influência do mar na primeira área, ao contrário da segunda, que por ser mais interna está sob maior influência de águas continentais.

A densidade média de $U$. cordatus na Baía da Babitonga (Santa Catarina) foi menor quando comparada à de manguezais da região nordeste do Brasil (4-5 ind./ $\mathrm{m}^{2}$, segundo AlCÂntaraFilho 1978, Costa 1979 e Nascimento et al. 1982), embora seja pouco superior à dos manguezais da Baía das Laranjeiras (Paraná) (0,6-2,5 ind./m², segundo Blankensteyn et al. 1997). Tal fato é, provavelmente, resultante da maior similaridade entre os bosques de manguezal do Paraná e Santa Catarina, localizados no limite de distribuição austral deste ecossistema.

A menor densidade desse caranguejo na região sul brasileira pode estar relacionada à maior influência de frentes frias, que podem ser críticas ao desenvolvimento adequado da espécie. Tal aspecto foi considerado por BRANCO (1993), em manguezais de Florianópolis (Santa Catarina), onde menciona que a temperatura interna nas galerias chega a atingir valores inferiores a $15^{\circ} \mathrm{C}$. Segundo este autor a densidade média do caranguejo-uçá para os manguezais de Florianópolis (Santa Catarina) é de 2,2 galerias abertas $/ \mathrm{m}^{2}$, similar ao obtido no presente estudo. Desta forma, a variação latitudinal pode ser um fator preponderante, podendo atuar na limitação ou mesmo restrição de eventos biológicos e reprodutivos de uma espécie em determinadas épocas do ano (Henmi 1993).

A Baía da Babitonga é um dos poucos remanescentes de manguezal da região sul do Brasil. Tal fato, associado à posição econômica de destaque dessa espécie e seu uso como recurso de subsistência para a população local, vem refletir a importância do presente estudo. Certamente, futuras informações sobre a biologia e ecologia do caranguejo-uçá permitirão o manejo mais adequado deste item pesqueiro, em especial para a Baia da Babitonga (Santa Catarina), que tem neste trabalho uma primeira contribuição.

\section{AGRADECIMENTOS}

Ao Centro de Pesquisa e Gestão de Recursos Pesqueiros do Litoral Sudeste e Sul, pelo financiamento da pesquisa; à Secretaria Municipal de Agricultura e Pesca de São Francisco do Sul; à Colônia de Pescadores Z-02, representada pelo seu então presidente Artur Carvalho; à Universidade da Região de Joinville; aos membros do Grupo de Pesquisa em Biologia de Crustáceos que auxiliaram nas coletas de campo e análises de laboratório (Gustavo Y. Hattori, Ronaldo A. Christofoletti e Ana G.
Fiscarelli); a Roberto F.C. Fontes (UNESP/CLP) pelo auxílio com o resumo em inglês; e aos catadores e pescadores, pois sem eles a realização desse projeto não teria sido possível.

\section{LITERATURA CITADA}

ADAmczewsKa, A.M. \& S. Morris. 2001. Ecology and behavior of Gecarcoidea natalis, the Christmas Island red crab, during the annual breeding migration. Biological Bulletin 200 (3): 305-320.

Alcântara-Filho, P. 1978. Contribuição ao estudo da biologia e ecologia do caranguejo-uçá, Ucides cordatus (Linnaeus, 1763) (Crustacea, Decapoda, Brachyura) no manguezal do Rio Ceará (Brasil). Arquivos de Ciências do Mar 18 (1/2): 1-41.

Alves, R.R.N. \& A.K. Nishida. 2004. Aspectos socioeconômicos e percepção ambiental dos catadores de caranguejo-uçá Ucides cordatus (L., 1763) (Decapoda, Brachyura) do estuário do Rio Mamanguape, nordeste do Brasil. Interciência 28 (1): 36-43.

Alves, R.R.A.; A.K. Nishida \& M.I.M. Hernández. 2005. Environmental perception of gatherers of the crab "caranguejo-uçá" (Ucides cordatus, Decapoda, Brachyura) affecting their collection attitudes. Journal of Ethnobiology and Ethnomedicine 1 (10): 1-8.

Amouroux, J.M. \& M. TAVAres. 2005. Natural recovery of Amazonian mangrove Forest as revealed by brachyuran crab fauna: preliminary description. Vie et Milieu-Life and Environment 55 (2): 71-79.

Blankensteyn, A.; D. Cunha-Filho \& A.S. Freire. 1997. Distribuição dos estoques pesqueiros e conteúdo protéico do caranguejo do mangue Ucides cordatus (L. 1763) (Brachyura, Ocypodidae) nos manguezais da Baía das Laranjeiras e Adjacências, Paraná, Brasil. Arquivos de Biologia e Tecnologia 40 (2): 331-349.

BuIss, D.E. 1990. Shrimps, Lobsters and Crabs. New York, Columbia University Press, 242p.

Branco, J.O. 1993. Aspectos ecológicos do caranguejo Ucides cordatus (Linnaeus, 1763) (Crustacea, Decapoda) do manguezal do Itacorubi, Santa Catarina, Brasil. Arquivos de Biologia e Tecnologia 36 (1): 133-148.

BREITfuss, M.J. 2003. Defining the characteristic of burrows to better estimate abundance the grapsid crab, Helograpsus haswellianus (Decapoda, Grapsidae), on East Australian salt marsh. Crustaceana 76 (4): 499-507.

Clark, M.E.; T.G. Wolcott; D.L. Wolcott \& A.H. Hines. 1999. Foraging and agonistic activity co-occur in free-ranging blue crabs (Callinectes sapidus): observation of animals by ultrasonic telemetry. Journal of Experimental Marine Biology and Ecology 233 (1): 143-160.

Conde, J.E.; M.M.P. Tognella; E.T. Paes; M.L.G. Soares; I.A. LouRo \& Y. Schaeffer-Novelli. 2000. Population and life history features of the crab Aratus pisonii (Decapoda: Grapsidae) in a subtropical estuary. Interciência 25 (3): 151-158.

Costa, R.S. 1979. Bioecologia do caranguejo-uçá, Ucides cordatus 
(Linnaeus, 1763) Crustacea, Decapoda - no nordeste brasileiro. Boletim da Sociedade Cearense de Agronomia 20: $1-74$.

Crane, J. 1943. Display, breeding and relationships of fiddler crabs (Brachyura, genus $U c a$ ), in the northeastern United States. Zoologica 28 (23): 217-223.

Dalabona, G. \& J. Loyola-Silva. 2005. Período reprodutivo de Ucides cordatus (Linnaeus) (Brachyura, Ocypodidae) na Baía das Laranjeiras, sul do Brasil. Acta Biológica Paranaense 34: $115-126$.

Debelius, H. 1999. Crustacea Guide of the World. Frankfurt, Ikan, 321p.

Diele, K. 2000. Life history and population structure of the exploited mangrove crab Ucides cordatus (Linnaeus, 1763) (Decapoda: Brachyura) in the Caeté Estuary, North Brazil. Bremen, Center for Tropical Marine Ecology, Contribution 9, ZMT Bremen, 103p.

Diele, K.; V. Koch \& U. SAint-Paul. 2005. Population structure, catch composition and CPUE of the artisanally harvested mangrove crab Ucides cordatus (Ocypodidae) in the caeté estuary, North Brazil: Indications for overfishing? Aquatic Living Resource 18: 169-178.

Fausto-Filho, J. 1968. Crustáceos decápodos de valor comercial ou utilizados como alimento no nordeste brasileiro. Boletim da Sociedade Cearense de Agronomia 9: 27-28.

Fiscarelli, A.G. \& M.A.A. Pinheiro. 2002. Perfil sócio-econômico e conhecimento etnobiológico do catador do caranguejo-uçá, Ucides cordatus (Linnaeus, 1763) nos manguezais de Iguape (244ㄴ), SP, Brasil. Actualidades Biologicas 24 (77): 129-142.

Flores, A.V.V.; K.G. Abrantes \& J. Paula. 2005. Estimating abundance and spatial distribution patterns of the bubble crab Dotilla fenestrata (Crustacea, Brachyura). Austral Ecology 30 (1): 14-23.

Geraldes, M.G. \& I.B. Calventi. 1983. Estudios experimentales para el mantenimiento en cautiverio del cangrejo Ucides cordatus. Ciência Interamericana 23 (4): 41-53.

Glaser, M. \& K. Diele. 2004. Asymmetric outcomes: Assessing central aspects of the biological, economic and social sustainability of a mangrove crab fishery, Ucides cordatus (Ocypodidae), in North Brazil. Ecological Economics 49 (3): 361373.

Guest, M.A.; R.M. Connolly; S.Y. Lee; N.R. Loneragan \& M.J. BReITfuss. 2006. Mechanism for the small-scale movement of carbon among estuarine habitats: Organic matter transfer not crab movement. Oecologia 148: 88-96.

Haley, S.R. 1972. Reproductive cycling in the ghost crab, Ocypode quadrata (Fabr.) (Brachyura, Ocypodidae). Crustaceana 23 (1): 1-11.

Hartnoll, R.G. 1969. Mating in the Brachyura. Crustaceana 16: 161-181.

Henmi, Y. 1989. Life-history patterns in two forms of Macrophthalmus japonicus (Crustacea, Brachyura). Marine Biology
101 (1): 53-60.

Henmi, Y. 1993. Geographic variations in life-history traits of the intertidal ocypodid crab Macrophthalmus banzai. Oecologia 96: 324-330.

Henmi, Y. \& M. Kaneto. 1989. Reproductive ecology of three ocypodid crabs I. The influence of activity differences on reproductive traits. Ecological Research 4 (1): 17-29.

IвAмA. 1994. Lagosta, Caranguejo-Uçá e Camarão-do-Nordeste. Brasília, Coleção Meio Ambiente, Série Estudos-Pesca, vol. 10, 190p.

IвAмA. 1998. Proteção e controle de ecossistemas costeiros: manguezal da Baía de Babitonga. Brasília, Coleção meio ambiente, Série Estudos-Pesca, vol. 25, 146p.

Ivo, C.T.C.; A.F. Dias; E.R.O. Botelho; R.I. Mota; J.A. VasconceLos \& E.M.S. VAsconcelos. 2000. Caracterização das populações de caranguejo-uçá, Ucides cordatus (Linnaeus, 1763), capturados em Estuários do Nordeste do Brasil. Boletim Técnico-Científico do CEPENE 8: 9-43.

Jankowsky, M.; J.S.R. Pires \& N. Nordi. 2006. Contribuição ao manejo participativo do Caranguejo-uçá, Ucides cordatus (L.) 1763), em Cananéia, SP. Boletim do Instituto de Pesca 32 (2): 221-228.

JoRdÃo, J.M. \& R.F. OliveIRA. 2003. Comparision of non-invasive methods for quantifying population density of the fiddler crab Uca tangeri. Journal of the Marine Biological Association of United Kingdom 83 (5): 981-982.

Koolkalya, S.; T. Thapanand; S. Tunkijuanujij; V. Havanont \& T. JutAGATE. 2006. Aspects in spawning biology and migration of the mud crab Scylla olivacea in the Andaman Sea, Thailand. Fisheries Management and Ecology 13: 391-397.

Күомо, J. 1988. Analysis of the relationship between gonads and hepatopancreas in males and females of the crab Sesarma intermedia, with reference to resource use and reproduction. Marine Biology 97 (1): 87-93.

Lindley, J.A. 1990a. Regressions for estimating development in the shrimp, Palaemonetes pugio Holthuis (Caridea, Palaemonidae). Crustaceana (Suppl. 2): 19-26

Lindley, J.A. 1990b. Regressions for estimating development times of the pelagic larvae of Paguridae and Porcellanidae. Journal of Plankton Research 12 (3): 673-678.

Lourenço, R.; J. Paula \& M. Henrique. 2000. Estimating the size of Uca tangeri (Crustacea, Ocypodidae) without massive crab capture. Scientia Marina 64 (4): 437-439.

Macia, A.; I. Quincardete \& J. Paula. 2001. A comparison of alternative methods for estimating population density of the Uca annulipes at Saco Mangrove, Inhaca Island (Mozambique). Hydrobiologia 449: 213-219.

Macintosh, D.J. 1988. The ecology and physiology of decapods of mangrove swamps. Symposium Zoological Society London 59: 315-341.

Melo, G.A.S. 1996. Manual de identificação dos Brachyura (caranguejos e siris) do litoral brasileiro. São Paulo, Ed. Plêiade/FAPESP, 604p. 
Morgan, S.G. 1996. Influence of tidal variation on reproductive timing. Journal Experimental Marine Biology and Ecology 206: 237-251.

Morgan, S.G. \& J.H. Christy. 1996. Adaptive significance of the timing of larval release by crabs. American Naturalist 145: 457-479.

Mota-Alves, M.I. 1975. Sobre a reprodução do caranguejo-uçá, Ucides cordatus (Linnaeus), em mangues do Estado do Ceará - Brasil. Arquivos de Ciências do Mar 15 (2): 85-91.

NASCimento, S.A. 1993. Biologia do Caranguejo-Uçá (Ucides cordatus). Sergipe, Administração Estadual do Meio Ambiente (ADEMA), 45p.

Nascimento, S.A.; E.R. Santos; L. Bonfim \& R.S. Costa. 1982. Estudo Bio-Ecológico do Caranguejo Uçá Ucides cordatus. Sergipe, Administração Estadual do Meio Ambiente, 12p.

Nobbs, M. \& K. Mcguiness. 1999. Developing methods for quantifying the apparent abundance of fiddler crabs (Ocypodidae: Uca) in mangrove habitats. Australian Journal Ecology 24: 43-49.

Nordhaus, I.; M. WolfF \& K. Diele. 2006. Litter processing and population food intake of the mangrove crab Ucides cordatus in a high intertidal forest in northern Brazil. Estuarine, Coastal and Shelf Science 67: 239-250.

NoRDI, N. 1994. A captura do caranguejo-uçá (Ucides cordatus) durante o evento reprodutivo da espécie: O ponto de vista dos caranguejeiros. Revista Nordestina de Biologia 9 (1): 41-47.

NyE, P.A. 1977. Reproduction, growth and distribution of the grapsid crab Helice crassa (Dana, 1851) in the southern part of New Zealand. Crustaceana 33 (1): 75-89.

Passos, C.A. \& A.P. DI BeneditTo. 2005. Aspectos da captura comercial do caranguejo-uça, Ucides cordatus (L., 1763), no manguezal de Gargaú, RJ. Biotemas 18 (1): 223-231.

Payen, G.G. 1980. Aspects fondamentaux de l'endocrinologie de la reproduction chez les crustacés marins. Oceanis 6 (3): 309-339.

Pinheiro, M.A.A. \& A.G. Fiscarelli. 2001. Manual de apoio à fiscalização do caranguejo-uçá (Ucides cordatus). Itajaí, Cepsul/IBAMA, 43p.

Submitted: 09.XI.2007; Accepted: 02.VI.2008.

Editorial responsibility: Georgina Bond-Buckup
Pinheiro, M.A.A. \& A. Fransozo. 2002. Reproduction of the speckled swimming crab Arenaeus cribrarius (Brachyura: Portunidae), in the southern coast of Brazil. Journal of Crustacean Biology 22 (2): 416-428.

Pinheiro, M.A.A.; A. Fransozo \& M.L. Negreiros-Fransozo. 1994. Estimativa da duração larval em função da temperatura para a Família Majidae (Crustacea, Decapoda, Brachyura). Boletim do Instituto de Pesca 21: 75-81.

Pinheiro, M.A.A. \& G.Y. Hattori. 2006. Relative growth of the mangrove crab Ucides cordatus (Crustacea, Brachyura, Ocypodidae) at Iguape (SP), Brazil. Brazilian Archives of Biology and Technology 49 (5): 813-823.

Rodrigues, A.M.T.; E.J. Branco; S.A. Saccardo \& A. Blankensteyn. 2000. A exploração do caranguejo Ucides cordatus (Decapoda: Ocypodidae) e o processo de gestão participativa para a normatização da atividade na Região Sudeste-Sul do Brasil. Boletim do Instituto de Pesca 26 (1): 63-78.

SASTRY, A.N. 1983. Ecological aspects of reproduction, p. 179270. In: F.J. Vernberg \& W.B Vernberg (Eds). The Biology of Crustacea. Environmental adaptations. New York, Academic Press, 383p.

Skov, M.W.; M. Vaninni; J.P. Shunula; R.G. Hartnoll \& S. Cannicci. 2002. Quantifying the density of mangrove crabs: Ocypodidae and Grapsidae. Marine Biology 141 (4): 725-732.

SKov, M.W. \& R.G. HaRTNoll. 2001. Comparative suitability of binocular observation, burrow counting and excavation for the quantification of the mangrove fiddler crab Uca annulipes (H. Milne Edwards). Hydrobiologia 449: 201-212.

SoKAL, R.R. \& J. RoнLf. 2003. Biometry: the principles and practice of statistics in biological research. New York, Freeman and Company, $3^{\text {rd }}$ ed., $887 \mathrm{p}$.

Warren, J.H. 1990. The use of open burrows to estimate abundance of intertidal estuarine crabs. Australian Journal of Ecology 15: 277-280.

Wolff, M.; V. Koch \& V. Isaac. 2000. A trophic flow model of the Caeté Mangrove Estuary (North Brazil) with considerations and for the sustainable use of its resources. Estuarine, Coastal and Shelf Science 50: 789-803. 